\title{
On Cauchy-Kowalevski's Theorem; A Necessary Condition
}

\author{
By
}

\author{
Sigeru Mizohata*
}

\section{Introduction}

We are concerned with the Cauchy-Kowalevski theorem for an equation

$$
\begin{gathered}
\partial_{t}^{m} u(x, t)=\sum_{j=1}^{m} a_{j}\left(x, t ; \partial_{x} \partial_{t}^{m-j} u(x, t)+f(x, t),\right. \\
(x, t) \in C^{l} \times C^{1},
\end{gathered}
$$

where the coefficients are assumed holomorphic in a neighborhood of the origin.**) The Cauchy-Kowalevski theorem says that, if

$$
\operatorname{order}\left(a_{j}\right) \leqq j \text {, }
$$

then for any holomorphic Cauchy data $\left.\partial_{t}^{\jmath} u\right|_{t=0}=u_{j}(x) \quad(0 \leqq j \leqq m-1)$, and for any holomorphic $f$, given in the neighborhood of the origin, there exists a unique holomorphic solution $u$ of (1.1) in a neighborhood of the origin. In (1.2), order $\left(a_{j}\right)$ means that of $a_{j}$ in a neighborhood of the origin. Our question is the following: Is the condition (1.2) necessary for the Cauchy-Kowalevski theorem? Concerning this, the author showed in [3] the following result. Let $q(>1)$ be the minimum number satisfying

$$
\operatorname{order}\left(a_{j}\right) \leqq q j \quad(1 \leqq j \leqq m) \text {, }
$$

and let $h_{j}(x, t ; \partial)$ be the homogeneous part of order $q j$ of $a_{j}$. Then in order that the above Cauchy-Kowalevski theorem hold, it is necessary that

Received March 30, 1974.

*) Department of Mathematics, Kyoto University, Kyoto.

**) We use the following abbreviations: $\partial_{x}^{\alpha}, \partial_{t}^{j}$ stand for $\left(\frac{\partial}{\partial x}\right)^{\alpha},\left(\frac{\partial}{\partial t}\right)^{j}$ respectively. Furthermore, $\partial_{x}^{\alpha}$ will be denoted simply by $\partial^{\alpha}$. 


$$
h_{j}(x, 0: \zeta) \equiv 0 .
$$

This implies, in particular, when all the terms of order greater than $j$ of $a_{j}(x, t ; \zeta)$ are independent of $t$, then $(1.2)$ becomes a necessary condition for the validity of the Cauchy-Kowalevski theorem.

Recently M. Miyake investigated this problem [2], and showed that in the case $m=1$, namely,

$$
\partial_{t} u=\sum_{j} b_{j}\left(x, t ; \partial_{x}\right) u+f, \quad\left(\operatorname{order}\left(b_{j}\right)=j\right),
$$

the condition $b_{j}(x, t ; \zeta) \equiv 0$ for $j \geqq 2$ is really necessary. So that $(1.2)$ is necessary and sufficient in the case $m=1$. The purpose of this article is to show that when we follow the argument of Miyake together with that of Hasegawa in [1], we arrive at a sharper result than (1.3). Let us explain this. We expand each $a_{j}(x, t ; \zeta)$ appearing in (1.1) in Taylor series in $t$ around the origin. Then the terms appearing on the right hand side take the form:

$$
t^{n} a(x) \partial_{t}^{\alpha} \partial_{t}^{j} \quad(a(x) \not \equiv 0) .
$$

To all these terms, we define $p$ (rational number) as the minimum satisfying

$$
|\alpha|+p(j-n) \leqq p m .
$$

By saying modified principal part with weight $p$ of (1.1), we mean all the terms for which the equal sign hold. Our result is:

Theorem. In order that the Cauchy-Kowalevski theorem hold at the origin, it is necessary that $p \leqq 1$. Accordingly, in particular,

$$
\operatorname{order}\left(a_{j}\left(x, 0 ; \partial_{x}\right)\right) \leqq j
$$

is a necessary condition.

\section{Preliminaries}

To make clear our argument, we treat (1.1) in matrix form. Put $\partial_{t}^{j} u=v_{j+1}(0 \leqq j \leqq m-1)$. Then (1.1) becomes

$$
\partial_{t} v(x, t)=P\left(x, t ; \partial_{x}\right) v(x, t)+g(x, t) .
$$


The Taylor expansion of $P\left(x, t ; \partial_{x}\right)$ in $t$ gives

$$
P\left(x, t ; \partial_{x}\right)=\sum_{j=0}^{\infty} t^{j} P_{j}\left(x ; \partial_{x}\right)
$$

where

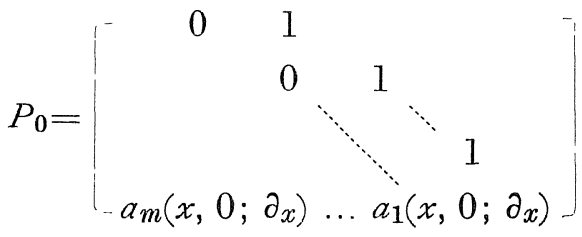

$$
\begin{aligned}
& P_{j}=\left[\begin{array}{c}
0 \\
a_{j m}\left(x ; \partial_{x}\right) \ldots a_{j 1}\left(x ; \partial_{x}\right)
\end{array}\right] \quad(j \geqq 1)
\end{aligned}
$$

From the definition of the number $p$, we have

$$
\operatorname{order}\left(a_{j k}(x ; \partial)\right) \leqq p j+p k \quad(j \geqq 0,1 \leqq k \leqq m) \text {. }
$$

Our purpose is to show that, assuming $p>1$, a formal solution corresponding to an appropriate holomorphic $f$ does not converge in any neighborhood of the origin (assuming the initial data is 0 ).

Let the modified principal part (with weight $p$ ) of $P_{j}$ be $\stackrel{\circ}{P}_{j}(x ; \partial)$. It is easy to see that there exists an $s$ such that $\stackrel{\circ}{P}_{s} \neq 0$ but $\stackrel{\circ}{P}_{j}=0$ for $j>s$. For the foregoing argument, $\stackrel{\circ}{P}_{s}$ plays an important role. The case $s=0$ can be treated in the similar way as in [3]. Therefore we suppose $s \geqq 1$.

Let

$$
P_{s}(x ; \partial)=\left[\begin{array}{c}
0 \\
h_{s 1}(x ; \partial) \ldots h_{s m}(x ; \partial)
\end{array}\right]
$$

and $h_{s 1}(x ; \partial) \equiv \ldots \equiv h_{s, i-1}(x ; \partial) \equiv 0$. but $h_{s i}(x ; \partial) \not \equiv 0$, Then, as we shall show at the end of this section, if $h_{s i}(0 ; \zeta) \equiv 0$, then by choosing $x_{0}$ near the origin such that $h_{s i}\left(x_{0} ; \zeta\right) \neq 0$, without loss of generality, we can assume that

$$
h_{s i}(0 ; \zeta) \neq 0, \text { for } \zeta \in C^{l} \quad(|\zeta|=1)
$$

We fix such a $\zeta$ once for all. 
Next, by choosing $j_{0}$ satisfying $p j_{0}=$ integer, we take $f(x, t)$ as

$$
\left\{\begin{array}{l}
f(x, t)=\frac{t^{j_{0}}}{j_{0} !} f_{0}(x), \\
f_{0}(x)=\Sigma_{k}^{\prime} c_{k} \frac{\langle\zeta, x\rangle^{p k}}{(p k) !}
\end{array}\right.
$$

where $\Sigma_{k}^{\prime}$ means the summation over all positive integers $k$ satisfying $p k=$ integer; $c_{k}$ will be defined appropriately. To be precise, $\left|c_{k}\right|=$ $(p k)$ ! and their arguments are defined recursively. So that $f_{0}(x)$ is holomorphic in $|x|<1$.

Let

$$
v(x, t) \sim \sum_{j=0}^{\infty} \frac{t^{j}}{j !} v_{j}(x)
$$

be the corresponding formal solution. We assume that $v_{0}(x) \equiv 0$, i.e. Cauchy data is zero. Then denoting

$$
g_{0}(x)={ }^{t}\left(0,0, \ldots, 0, f_{0}(x)\right),
$$

we have $v_{0}(x) \equiv v_{1}(x) \equiv \ldots \ldots \equiv v_{j_{0}}(x) \equiv 0$. Comparing the coefficient of $t^{n-1}$, we get

$$
\left\{\begin{array}{l}
v_{n}(x)=P_{0}(x ; \partial) v_{n-1}(x)+(n-1) P_{1}(x ; \partial) v_{n-2}(x)+ \\
+(n-1)(n-2) P_{2}(x ; \partial) v_{n-3}+\ldots+(n-1) \ldots(n-s) P_{s}(x ; \partial) v_{n-s-1}+\ldots
\end{array}\right.
$$

Let us remark that

$$
v_{j+1}(x)=P_{0}(x ; \partial) g_{0}(x)
$$

Finally our observation mentioned above (see the assumption with regards to $h_{s i}$ ) relies on the following proposition which we used in [3]:

Proposition. Let $\mathcal{O}$ be a complex domain (in $C^{l+1}$ ) containing the origin. We assume all the coefficients of (1.1) belong to $H(\mathcal{O})$, i.e. holomorphic in $\mathcal{O}$. Assume for each $f(x, t) \in H(\mathcal{O})$ there exists a solution $u(x, t) \in H\left(V_{f}\right)$ of (1.1) satisfying

$$
\partial_{t}^{j} u(x, 0)=0 \text { for } x \in V_{f} \cap\{t=0\} \quad(0 \leqq j \leqq m-1),
$$

where $V_{f}$ is a complex domain containing the origin which may depend 
on $f$. Then there exists a fixed complex domain $D$ containing the origin such that for any $f \in H(\mathcal{O})$, there exists always a solution $u(x, t) \in H(D)$ of (1.1) with zero Cauchy data.

Proof. Let

$$
D_{m}=\left\{(x, t) \in C^{l+1} ;\left|x_{i}\right|<1 / m,|t|<1 / m\right\} .
$$

For any pair $(m, n)$ of positive integers, we define the set $E_{m n} \subset H(\mathcal{O})$ as follows. $f \in E_{m n}$, if and only if there exists a solution $\iota(x, t) \in H\left(D_{m}\right)$ of (1.1) with zero Cauchy data, satisfying

$$
|u(x, t)| \leqq n, \text { for }(x, t) \in D_{m} .
$$

Then $E_{m n}$ is closed and symmetric. In fact, if $\left\{f_{j}\right\}$ is a sequence of $E_{m n}$, and $f_{j} \rightarrow f_{0}$ in $H(\mathcal{O})$, and let $\left\{u_{j}\right\}$ be the corresponding solution. If necessary, by picking a subsequence, we can assume $\left\{\imath \iota_{p}(x, t)\right\}$ is a convergent sequence in $H\left(D_{n}\right)$. Then $u_{j_{p}}(x, t) \rightarrow u_{0}(x, t)$ in $H\left(D_{m}\right)$, and $\left|u_{0}(x, t)\right| \leqq n$ in $D_{m}$. Since

$$
L\left(u_{j_{p}}\right) \equiv \partial_{t}^{m} u_{j_{p}}(x, t)-\sum a_{j}\left(x, t ; \partial_{x}\right) \partial_{t}^{m-j} u_{j_{p}}(x, t)
$$

tends to $L\left(u_{0}\right)$ in $H\left(D_{m}\right)$, we obtain $L\left(u_{0}\right)=f_{0}$ which proves the closedness of $E_{m n}$. In view of $H(\mathcal{O})=\cup_{m, n} E_{m n}$ by hypothesis, the proposition follows immediately from Baire's category theorem. $\quad$ Q.E.D.

Now let us make precise our hypothesis. First, let

$$
\mathcal{O}=\left\{(x, t) ;\left|x_{i}\right|<\rho,|t|<\rho\right\}
$$

where it is assumed $\rho<1 / 2 l$ (recall that $l$ is the dimension of $x$-space). Then, from the above proposition, we can find a polydisc $D$, say $D=$ $\left\{(x, t) ;\left|x_{i}\right|<\rho_{0},|t|<\rho_{0}\right\}$. Now when $h_{s i}(0 ; \zeta) \equiv 0$ for all $\zeta$, and $h_{s i}(x ; \zeta) \neq 0$, we can find $x_{0}\left(\in \mathbb{R}^{l}\right)$ in such a way that denoting $x_{0}=$ $\left(x_{1}^{0}, \ldots, x_{n}^{0}\right)$, it satisfies $\left|x_{i}^{0}\right|<\min \left(\rho_{0}, 1 / 2 l\right)$, so that we have

$$
\mathcal{O} \subset \mathcal{O}^{\prime}=\left\{(x, t) ;\left|x_{i}-x_{i}^{0}\right|<1 / l,|t|<1 / l\right\},
$$

and that $\left(x_{1}^{0}, \ldots, x_{n}^{0}, 0\right) \in D$. This shows that

$$
f_{0}(x)=\Sigma_{k}^{\prime} c_{k} \frac{\left\langle\zeta, x-x_{0}\right\rangle^{p k}}{(p k) !} \quad\left(\left|c_{k}\right|=(p k) !\right)
$$


which is holomorphic in $\mathcal{O}^{\prime}$, is so in $\mathcal{O}$. Thus by hypothesis, the corresponding solution should be holomorphic in a neighborhood $V(\supset D)$ of $\left(x_{0}, 0\right)$.

\section{Proof of Theorem}

In (2.6), instead of $f_{0}(x)$ itself, we take simply

$$
f_{0}(x)=\frac{\langle\zeta, x\rangle^{p k}}{(p k) !} \quad(p k=\text { integer })
$$

and consider the coefficients $v_{j}(x)\left(j_{0} \leqq j \leqq j_{0}+k\right)$ defined by (2.8). We are concerned with the leading term, i.e. the lowest homogeneous part in $x$ of the $h$-th component $v_{j, h}(x)$ of $v_{j}(x)$. Note the following fact. Let

$$
\begin{aligned}
p(x, \partial) & =\sum_{|\alpha| \leqq n} a_{\alpha}(x) \partial^{\alpha}=\sum_{\mid \alpha=n} a_{\alpha}(x) \partial^{\alpha}+\sum_{|\alpha|<n} a_{\alpha}(x) \partial^{\alpha} \\
& =p_{n}(x, \partial)+q(x, \partial) .
\end{aligned}
$$

Then for $j \geqq n$,

$$
p(x, \partial) \frac{\langle\zeta, x\rangle^{j}}{j !}=p_{n}(0, \zeta) \frac{\langle\zeta, x\rangle^{j-n}}{(j-n) !}+\ldots \ldots
$$

where the rest term on the right hand side is analytic function of vanishing order $\geqq j-n+1$. In view of this, we see that

$$
v_{i, h}(x)=a_{j, h}\langle\zeta, x\rangle^{\nu(j, h)} / \nu(j, h) !+\ldots \ldots
$$

where $\nu(j, h)=p k-p\left(j-j_{0}\right)+p(m-h)$, and the rest term is of vanishing order $\geqq \nu(j, h)+1$.

Taking account of that, (2.8) gives a recurrence formula for $\alpha_{j, h}$. In fact, let

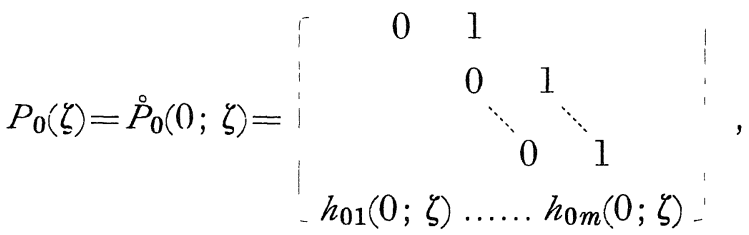

$$
\begin{aligned}
& P_{j}(\zeta)=\stackrel{\circ}{P}_{j}(0 ; \zeta)=\left[\begin{array}{c}
0 \\
h_{j 1}(0 ; \zeta) \ldots . . h_{j m}(0 ; \zeta)
\end{array}\right](j \geqq 1),
\end{aligned}
$$


where $h_{j k}(0 ; \zeta)$ are the terms of the modified principal part with weight $p$ (see the definition in the Introduction), and let

$$
\alpha_{n}={ }^{t}\left(\alpha_{n, 1}, \ldots, \alpha_{n, m}\right) \text {. }
$$

Then

$$
\left\{\begin{aligned}
\alpha_{n}= & P_{0}(\zeta) \alpha_{n-1}+(n-1) P_{1}(\zeta) \alpha_{n-2}+(n-1)(n-2) P_{2}(\zeta) a_{n-3}+ \\
& +\ldots+(n-1)(n-2) \ldots(n-s) P_{s}(\zeta) \alpha_{n-s-1}
\end{aligned}\right.
$$

where

$$
\alpha_{j}=0 \text { for } j<j_{0} \text { and } \alpha_{j_{0}}=t(0,0, \ldots, 0,1) \text {. }
$$

Hereafter we denote

$$
h_{s j}(0 ; \zeta)=h_{j} \quad(1 \leqq j \leqq m) .
$$

Then by hypothesis, $h_{1}=h_{2}=\ldots=h_{i-1}=0$, but $h_{i} \neq 0$. For convenience of the foregoing argument, let us denote

$$
\alpha_{n}^{\prime}={ }^{t}\left(\alpha_{n, i}, \alpha_{n, i+1}, \ldots, \alpha_{n, m}\right),
$$

and

$$
\left|a_{n}\right|=\sum_{j=1}^{m}\left|a_{n, j}\right| ; \quad\left|a_{n}^{\prime}\right|=\sum_{j=i}^{m}\left|a_{n, j}\right| \cdot
$$

Next we choose a $\delta(0<\delta<1)$ once for all in such a way that

$$
\max _{j>i}\left|h_{j}\right| \frac{\delta}{1-\delta} \leqq \frac{1}{2}\left|h_{i}\right|
$$

Then for the sequence $\left\{\alpha_{n}\right\}$ we have the following lemma:

Lemma. If $j_{0}$ is chosen large, then there exists an infinite subsequence $\left\{a_{n_{p}}\right\}$ of $\left\{\alpha_{n}\right\}$ ( $n \geqq j_{0}$ ) satisfying the following increasing law (in the wider sense):

$$
\left|a_{n_{p}}^{\prime}\right| \geqq \delta\left|a_{n_{p-1}}^{\prime}\right| \quad(p=2,3, \ldots)
$$

moreover we can assume that

$$
n_{p}-n_{p-1} \leqq s+1
$$


Proof. To define $\left\{n_{p}\right\}$, we proceed as follows: If $n_{p}$ is defined, then $n_{p+1}$ is defined as the minimum number $m\left(>n_{p}\right)$ satisfying

$$
\left|\alpha_{m}^{\prime}\right| \geqq \delta\left|\alpha_{n_{p}}^{\prime}\right|
$$

Accordingly, let $n_{p}=n$, then it suffices to prove the following fact: If

$$
\left|\alpha_{n+1}^{\prime}\right|,\left|\alpha_{a_{\top 2}}^{\prime}\right|, \ldots,\left|\alpha_{n+s}^{\prime}\right|<\delta\left|\alpha_{n}^{\prime}\right|,
$$

then,

$$
\left|\alpha_{n+s+1}^{\prime}\right| \geqq \delta\left|\alpha_{n}^{\prime}\right|
$$

Let us consider the last, i.e. the $m$-th component of $\alpha_{n+s+1}$. In view of (3.1), it amounts to consider those of $P_{s-i}(\zeta) a_{n+i}(0 \leqq i \leqq s)$. As we can observe, the case where $i=m$ is easy, so we argue as $i<m$. Denote the $m$-th component of $P_{s}(\zeta) \alpha_{n}$ by $\left(P_{s} \alpha_{n}\right)_{m}$.

$$
\left(P_{s}(\zeta) \alpha_{n}\right)_{m}=h_{i} \alpha_{n, i}+\sum_{j>i} h_{j} \alpha_{n, j}
$$

Thus,

$$
\left|\left(P_{s}(\zeta) \alpha_{n}\right)_{m}\right| \geqq\left|h_{i}\right|\left|\alpha_{n, i}\right|-\max _{j>i}\left|h_{j}\right| \sum_{j>i}\left|\alpha_{n, j}\right|
$$

On the other hand, from (2.8) we see that, for general $n$,

$$
\alpha_{n, j}=\alpha_{n-1, j+1} \quad(1 \leqq j \leqq m-1) .
$$

In fact, all the entries of $P_{1}(\zeta), \ldots, P_{s}(\zeta)$ are zero except the mth row, so that the components $\alpha_{n, j}(1 \leqq j \leqq m-1)$ of $\alpha_{n}$ can be defined simply by $a_{n}=P_{0}(\zeta) a_{n-1}$. By hypothesis (3.4),

$$
\sum_{j=i}^{m}\left|\alpha_{n+1, j}\right|<\delta \sum_{j=i}^{m}\left|\alpha_{n, j}\right|
$$

Next, by (3.6),

$$
\begin{gathered}
\sum_{j=i+1}^{m}\left|\alpha_{n, j}\right|=\sum_{j=i+1}^{m}\left|\alpha_{n+1, j-1}\right|=\sum_{j=i}^{m-1}\left|\alpha_{n+1, j}\right| \\
\leqq \sum_{j=i}^{m}\left|\alpha_{n+1, j}\right|<\delta \sum_{j=i}^{m}\left|\alpha_{n, j}\right| .
\end{gathered}
$$

Thus, 


$$
(1-\delta) \sum_{j=i+1}^{m}\left|\alpha_{n, j}\right|<\delta\left|\alpha_{n, i}\right|
$$

This implies, from (3.5) and (3.2),

$$
\left|\left(P_{s}(\zeta) \alpha_{n}\right)_{m}\right| \geqq\left|h_{i}\right|\left|\alpha_{n, i}\right|-\max _{j>i}\left|h_{j}\right| \frac{\delta}{1-\delta}\left|\alpha_{n, i}\right| \geqq \frac{1}{2}\left|h_{i}\right|\left|\alpha_{n, i}\right|
$$

Further, since

$$
\left|\alpha_{n}^{\prime}\right|=\left|\alpha_{n, i}\right|+\sum_{j=i}\left|\alpha_{n, j}\right| \leqq \mid \alpha_{n, i}\left(1+\frac{\delta}{1-\delta}\right),
$$

i.e. $\left|a_{n, i}\right| \geqq(1-\delta)\left|a_{n}^{\prime}\right|$, the above relation can be written as

$$
\left|\left(P_{s}(\zeta) \alpha_{n}\right) m\right| \geqq \frac{1-\delta}{2}\left|h_{i}\right|\left|\alpha_{n}^{\prime}\right|
$$

Finally let us consider the last component of $P_{s-1}(\zeta) a_{n+1}, P_{s-2}(\zeta)$ $a_{n+2}, \ldots, P_{0}(\zeta) a_{n+s}$. First, from our process of choosing $\left\{n_{p}\right\}$ (defined at the beginning), we have

$$
\left|a_{n-j}^{\prime}\right| \leqq \frac{1}{\delta^{j}}\left|a_{n}^{\prime}\right| \quad(j=1,2, \ldots) .
$$

In fact, it holds that $\left|\alpha_{n p-1}^{\prime}\right| \leqq \frac{1}{\delta}\left|\alpha_{n_{p}}^{\prime}\right|,\left|\alpha_{n_{p-2}}^{\prime}\right| \leqq \frac{1}{\delta}\left|\alpha_{n_{p-1}}^{\prime}\right| \leqq \frac{1}{\delta^{2}}\left|\alpha_{n_{p}}^{\prime}\right|$, and so on. Further for $n_{q-1}<\nu<n_{q}$, it holds $\left|\alpha_{\nu}^{\prime}\right|<\left|\alpha_{n_{q}}^{\prime}\right|$.

Next, from (3.6), for any $h(1 \leqq h \leqq i-1)$, we have

$$
\alpha_{n+j, h}=\alpha_{n+j-1, h+1}=\alpha_{n+j-2, h+2}=\ldots .
$$

This gives together with (3.8)

$$
\left|a_{n+j, k}\right| \leqq \frac{1}{\delta^{m}}\left|a_{n}^{\prime}\right| \quad(0 \leqq j \leqq s)
$$

and this is of course true for any $k(1 \leqq k \leqq m)$ (see (3.4)).

Thus we have

$$
\sum_{\nu=1}^{s}\left|\left(P_{s-\nu}(\zeta) a_{n+\nu}\right) m\right| \leqq s i n \cdot \max _{i, j}\left|h_{i j}(0 ; \zeta)\right| \frac{1}{\delta^{m}}\left|\alpha_{n}^{\prime}\right| \equiv K\left|\alpha_{n}^{\prime}\right|
$$

where 


$$
K=\frac{s m}{\delta^{m}} \max _{i, j}\left|h_{i j}(0 ; \zeta)\right|
$$

Finally from (3.1), (3.7) and (3.10), we obtain

$$
\begin{gathered}
\left|\alpha_{n+s+1, m} \geqq(n+s)(n+s-1) \ldots(n+1)\right|\left(P_{s}(\zeta) \alpha_{n}\right) m \mid \\
\quad-(n+s) \ldots(n+2) \sum_{\nu=1}^{s}\left|\left(P_{s-\nu}(\zeta) \alpha_{n+\nu}\right) m\right| \\
\geqq(n+s) \ldots(n+1)\left\{\frac{1-\delta}{2}\left|h_{i}\right|\left|\alpha_{n}^{\prime}\right|-\frac{1}{n+1} K\left|\alpha_{n}^{\prime}\right|\right\} .
\end{gathered}
$$

Now we define $j_{0}$ in such a way that

$$
\frac{1-\delta}{4}\left|h_{i}\right| j_{0} \geqq \max (1, K) \text {. }
$$

Then we have $\left|\alpha_{n+s+1, m}\right| \geqq\left|\alpha_{n}^{\prime}\right|$. This completes the proof.

Q.E.D.

Let us return to (2.6). The above lemma gives the following result: there exist $i_{0}, j\left(0 \leqq i_{0} \leqq s, 1 \leqq j \leqq m\right)$ such that, if we write

$$
v_{n+j_{0}-i_{0}, j}(x)=c_{n} \beta_{n} \frac{\langle\zeta, x\rangle^{k}}{k !}+\varphi_{n}(x)+\psi_{n}(x),
$$

where $k=p i_{0}+p(m-j), \psi_{n}(x)$ being of vanishing order strictly greater than $k$, and $\varphi_{n}(x)$ is determined by $\left\{c_{i}\right\}$ for $i<n$, then, there exists an infinite subsequence of $n$ satisfying

$$
\left|\beta_{n}\right| \geqq \delta^{\prime n} \quad\left(\delta^{\prime}>0\right)
$$

for a fixed $\delta^{\prime}$.

Let us explain this. First we choose $i_{0}$ in such a way that there exists an infinite subsequence $\left\{n_{p}\right\}$ stated in the above lemma, which is congruent to $1-i_{0}$ modulo the denominator of $p$. Next, $j$ is chosen in such a way that, for this subsequence, say $\left\{a_{n_{p}}^{\prime}\right\}$, we have $\left|a_{n_{p}, j}^{\prime}\right| \geqq \frac{1}{m}\left|a_{n_{p}}^{\prime}\right|$. Further let us decompose ( $p n=$ integer),

$$
f_{0}(x)=c_{n} \frac{\langle\zeta, x\rangle^{p n}}{(p n) !}+\sum_{k<n} c_{k} \frac{\langle\zeta, x\rangle^{p k}}{(p k) !}+\sum_{k>n}^{\prime} c_{k} \frac{\langle\zeta, x\rangle^{p k}}{(p k) !}
$$

Since the correspondence

$$
f_{0}(x) \longrightarrow v_{n}(x)
$$


is linear (see (3.1)), for the study of the structure of $v_{n}(x)$ we can consider the three terms separately. Now we see easily that the part of $v_{n+j_{0}-i_{0}, j}(x)$ corresponding to the third term in the above decomposition, is of vanishing order $\geqq p i_{0}+p(m-j)+p$, hence this is greater than $k+1$.

Thus we obtain taking account of $|\zeta|=1$,

$$
\left.\langle\bar{\zeta}, \partial\rangle^{k} v_{n+j_{0}-i_{0}, j}(x)\right|_{x=0}=c_{n} \beta_{n}+\left.\langle\bar{\zeta}, \partial\rangle^{k} \varphi_{n}(x)\right|_{x=0} .
$$

Now we define $c_{n}$ by

$$
c_{n}=(p n) ! e^{i \theta_{n}}
$$

where $\theta_{n}$ is fixed in such a way that $e^{i \theta_{n}} \beta_{n}$ and $\left.\langle\bar{\zeta}, \partial\rangle^{k} \varphi_{n}(x)\right|_{x=0}$ have the same argument. It follows from (3.13) that for an appropriate subsequence of $n$ the left hand side of (3.14) is greater than, in absolute value, $(p n) ! \delta^{\prime n}$.

Now we return to the formal solution (2.7), and consider

$$
\left.\left.\langle\bar{\zeta}, \partial\rangle^{k} v(x, t)\right|_{x=0} \sim \sum_{n \geq 0} \frac{t^{n}}{n !}\langle\bar{\zeta}, \partial\rangle^{k} v_{n}(x)\right|_{x=0}
$$

There are infinitely many integers of the form $n+j_{0}-i_{0}$ such that their $j$-th component are greater than in absolute value

$$
\frac{1}{\left(n+j_{0}-i_{0}\right) !} \mid c_{n} \beta_{n \mid} \geqq \frac{(p n) !}{\left(n+j_{0}-i_{0}\right) !} \delta^{\prime n} \underset{n \rightarrow \infty}{\sim} A c_{0}^{n} n^{(p-1) n},
$$

where $A$ and $c_{0}$ are appropriate positive constants. Since $p>1$, the above series is never convergent for any $t(\neq 0)$. This completes the proof of Theorem in the Introduction.

\section{References}

[1] Haseagawa, Y., On the initial-value problems with data on a double characteristics, $J$. Math. Kyoto Univ. 11-2 (1971), 357-372.

[2] Miyake, M., A remark on Cauchy-Kowalevski's theorem, to appear.

[3] Mizohata, S., On Kowalevskian systems, Uspehi Mat. Nauk. 29, (1974), 213-227. 
